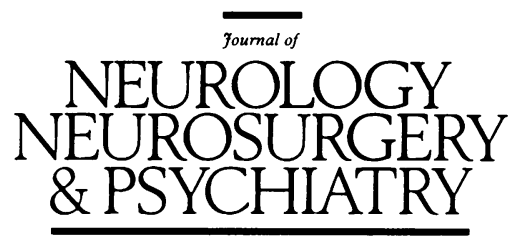

Editorial

\title{
Penfield's homunculus: a note on cerebral cartography
}

In 1937, Penfield and Boldrey published a paper of major importance. ${ }^{1}$ They described their work on the effects of stimulation of the cerebral cortex in man, the procedures being carried out as exploratory manoeuvres to delineate the appropriate area for subsequent surgical intervention. They confirmed the precise topography of cortical localisation, and were able to relate stimulation of a discrete part of the brain with motor and sensory phenomena affecting a particular part of the body. Whilst these now classical studies confirmed and greatly extended what had been known from earlier observations in awake humans and from experiments in animals, the manner of presentation of their findings was remarkable. It was the first time pictorial means of illustrating cortical representation had been attempted; it was thus an entirely new concept, but it was also one which has proved a curious method of illustration and one which gives rise to a number of unforseen problems.

Penfield and Boldrey set out to illustrate "the order and comparative extent" occupied in the sensorimotor strip. ${ }^{1}$ To represent the topography of their observations, the authors departed from the rigorous textual description of the effects of stimulation of the brain and achieved an extraordinary conceptual leap: an artist, Mrs H P Cantlie, was employed to draw a sensory and motor homunculus - a term discussed below. This first homunculus to be created (fig 1) was described as giving "a visual image of the size and the sequence of the cortical areas". It was symmetrical in shape and illustrated both motor and sensory features together; what actually was represented, however, was ambiguous and confusing. The authors state that the size of the parts was determined "not so much by the number of responses...but by the apparent perpendicular extent of representation of each part when these responses were multiple for the same part". What is meant by "perpendicular" is not stated, and it becomes even less clear from the subsequent comment: "that the large size of the thumb and the lips indicates the vertical extent of Rolandic cortex devoted to those parts in individual cases is very large". It is unclear whether "vertical" is the same as "perpendicular", and whether these terms mean longitudinal over the cortical surface, or deep-since the authors also stimulated deeper layers of the brain after having resected areas for extirpation of abnormal brain. The referral to responses which were multiple for the same part is also difficult to interpret, since it could imply multiple sites were effective in eliciting responses, or that only in cer- tain areas were multiple sites actually stimulated. Whilst the homunculus appears to relate to length rather than width, the parts illustrated are in fact enlarged in both dimensions. Also noteworthy is that responses have been transposed to the same side of the brain for the purposes of representation, and it is not possible from the illustration to distinguish unilateral from bilateral effects of cortical stimulation.

Thirteen years later, in their monograph entitled The cerebral cortex of man, Penfield and Rasmussen made another attempt to illustrate cortical representation. ${ }^{2}$ This was preceded by a diagram of a cross-section of the cere-

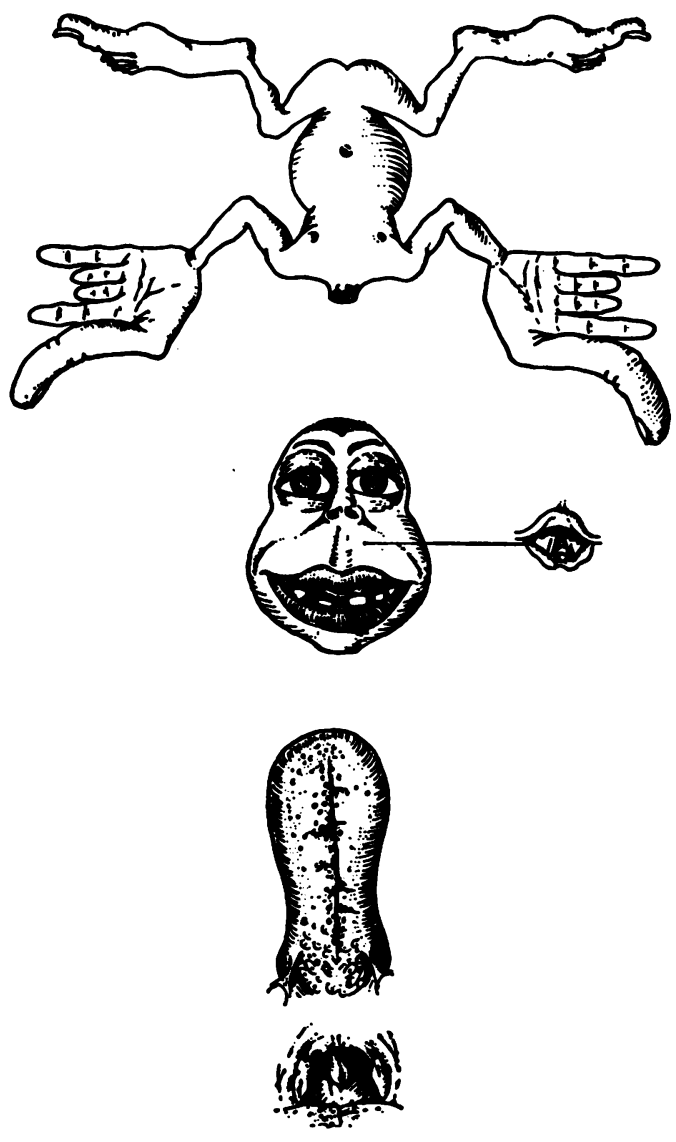

Figure 1 The first homunculus, Penfield and Boldrey, 1937. Reprinted from Brain 1937;60:389-443, with permission of The Macmillan Press Ltd. 
Figure 2 The motor and sensory homunculus: the first map. Penfield and Rasmussen, 1950. Reprinted with permission of Macmillan Publishing Company from The cerebral cortex of man by Wilder Penfield and Theodore Rasmussen. Copyright 1950 Macmillan Publishing

Company; copyright renewed 1978 Theodore Rasmussen.

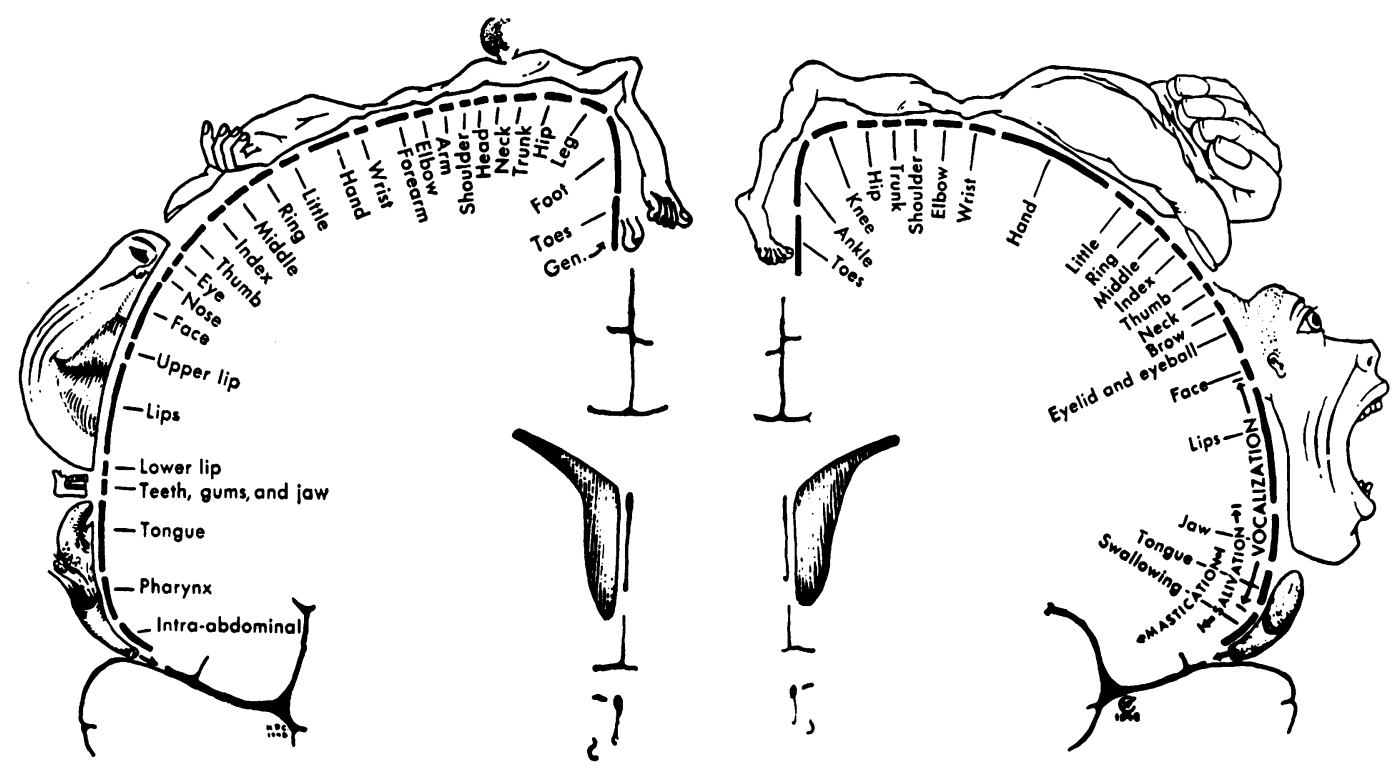

bral hemispheres in which were drawn solid bars at the periphery, the length of the bars giving an indication of the relative cortical areas from which the corresponding responses were elicited. This implies somewhat different information was being illustrated compared with the 1937 illustration. A few pages later, overlying the bars is superimposed the homunculus, which is again "brought forth" by the same artist, but in a different and even more familiar form, draped along the cortical surface and interhemispheric fissure. In this homunculus, however, the motor and sensory representations have been separated, slightly modified and corrected (fig 2). These drawings are so memorable and have been reproduced so often that it is difficult to appreciate that here, even more so than with the first homunculus, a new concept in representation and imagery had been created. Moreover, for the first time the homunculus can be considered as some form of "map" of human cortical representation, being more or less precisely in relation to actual brain areas identified at surgery. This compares with the first homunculus, which was not drawn in relation to the hemispheres at all. It is unclear whether the authors appreciated the visual significance of the homunculi, but these figurines created a precedent which has had a major influence on subsequent forms of related graphic illustration.

Degradation of the homunculus subsequently ensued, with the appearance in 1954 of multiple homunculi. Penfield and Jasper now illustrated three sets of homunculi (fig 3), the first set (motor and sensory) in relation to the Rolandic fissure, a second apparently purely sensory homunculus in the secondary sensory area near the Sylvian fissure, and a third homunculus (virtually only motor) in the supplementary motor cortex. ${ }^{3}$ The homunculi change considerably in this process; for instance the fingers and toes are made to seem important in the secondary sensory figurine, which is crouched, and is to some extent bilateral, whereas the supplementary homunculus is straight and extremely vaguely represented. The precision intended in the earlier homunculi has been almost completely lost; indeed Penfield and Jasper stated that "the exact position of the parts must not be considered topographically accurate. They are aids to memory, no more". And again, "the figurines...have the defects, and the virtues, of cartoons in that they are inaccurate anatomically...". It suggests that the homunculus has taken over from its authors, and illustration has out- stripped the scientific evidence available. Indeed, the bilateral leg movements that can be elicited by stimulation of medial structures led Bates to denigrate the concept of the homunculus thus: "May it not be, in other words, that in depicting the 'average' representation the motor homunculus should have two back legs?"4

Homunculi now appeared in other areas of the brain, such as the thalamus, and Penfield and Jasper ${ }^{3}$ were the first to place a homunculus in this subcortical region (fig 4 ). Whilst they stated a precise topographical organisation is present in the somatic relay nuclei of the thalamus, the homunculus drawn there "makes no pretence to

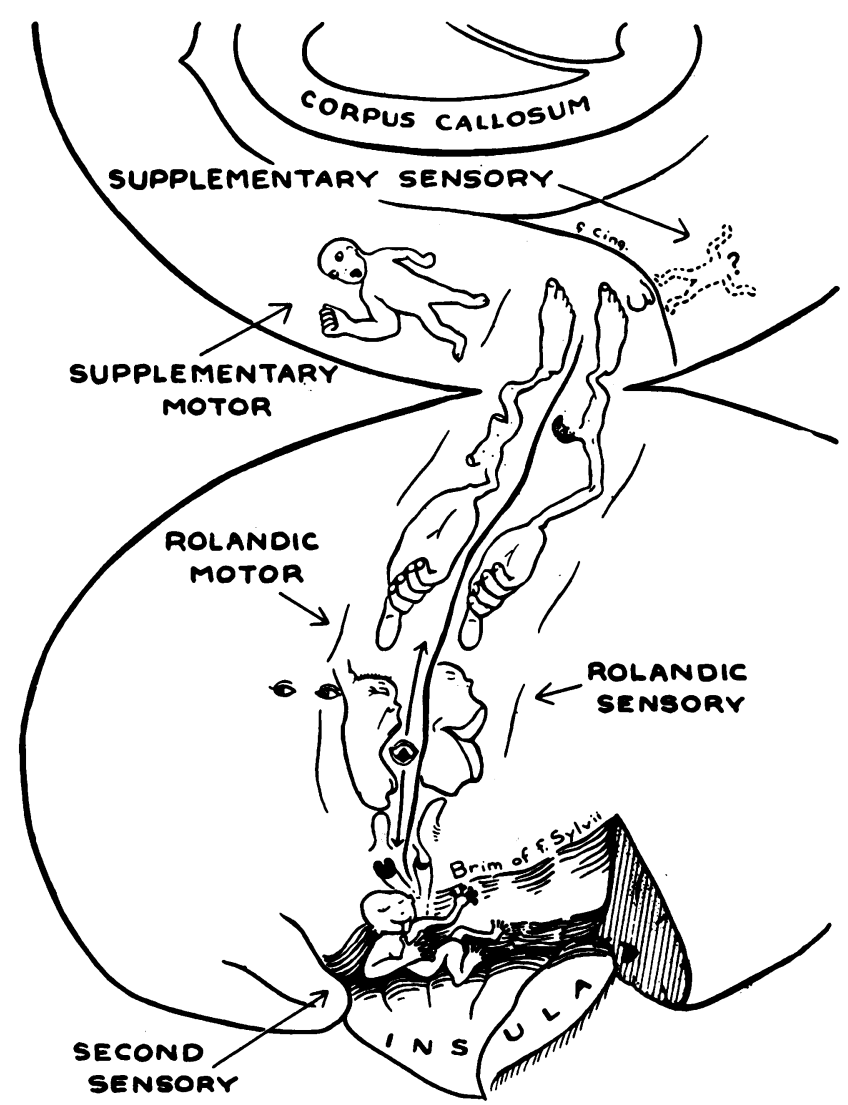

Figure 3 Mulltiple sets of homunculi. Penfield and fasper, 1954. Reprinted from Epilepsy and the functional anatomy of the human brain, with permission of Churchill Livingstone. 


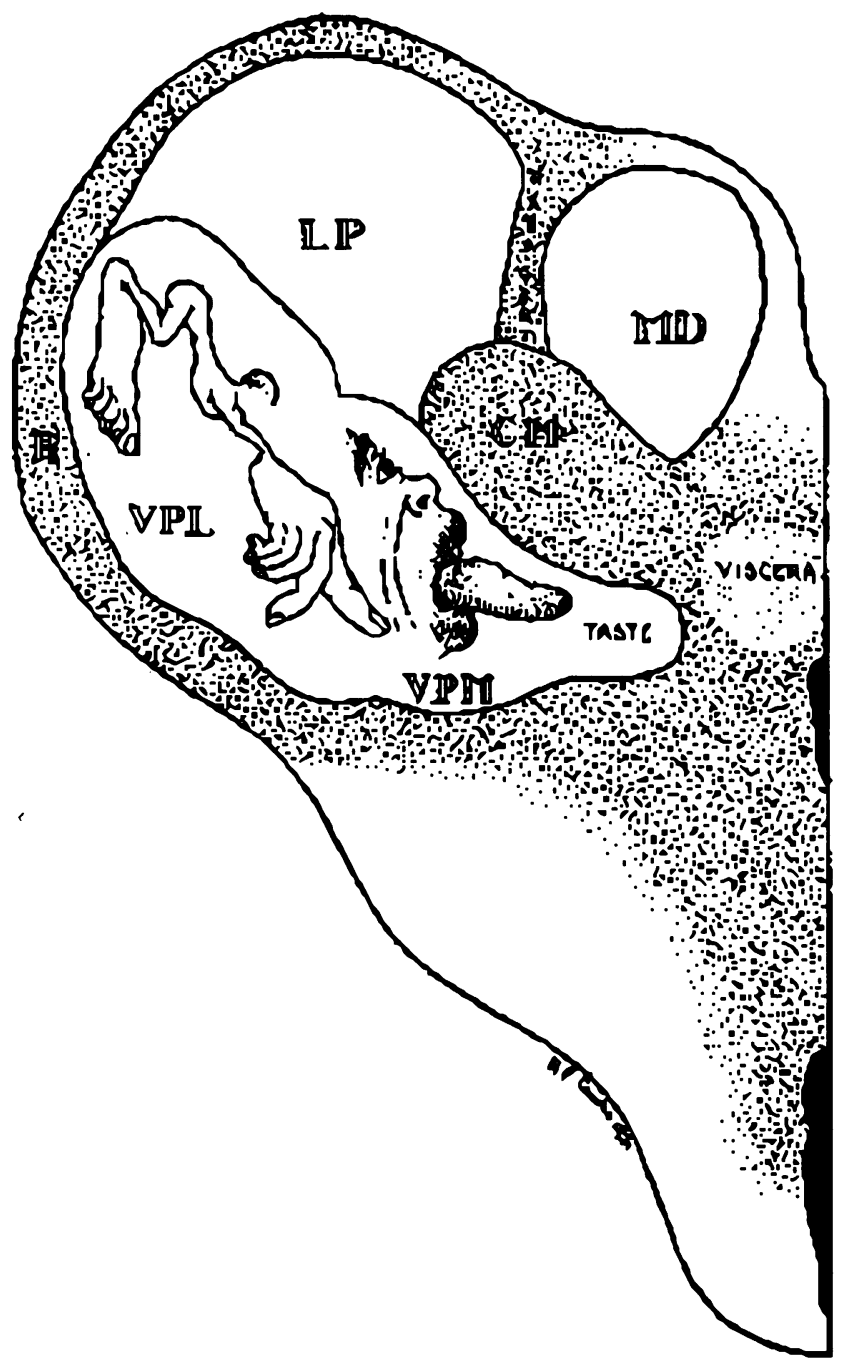

Figure 4 The homunculus in the thalamus. Penfield and fasper, 1954. Reprinted from Epilepsy and the functional anatomy of the human brain, with permission of Churchill Livingstone.

detailed accuracy, though the relationships between body parts are probably roughly correct, judging from animal experiments". The homunculus appears now to have degenerated into a purely artistic device vaguely based on animal experimental work, without any scientific basis to justify the human form portrayed. Final relegation to mere amusement must be the riot of homunculi with numerous images in the thalamic region depicted by Hassler et $a l^{5}$ and further annotated by Creutzfeldt. ${ }^{6}$ Whilst recognising the professional medical illustrator's role as an interpreter, ${ }^{7}$ any scientific significance of these displays is difficult to discern.

An interesting correspondence is referred to between Penfield and an un-named "distinguished neurologist", the latter pointing out that Hughlings Jackson had suggested the index finger would have a larger representation than the others; the discussion concludes by Penfield stating "the purpose of this monograph is to analyse the data, however imperfect they may be, without preconception". ${ }^{2}$ With this stated aim, however, it seems curiously contradictory to represent meticulous scientific observations by means of an homunculus. As implied by the preceding comments on Penfield and Jasper's multiple homunculi, even more than in the earlier homunculi, there is uncertainty as to what actually was being represented. Was it the surface area of the cortex, or the length or depth, or in addition some measure of the threshold of stimulation that produced motor or sensory effects?
Homunculi perhaps represent different things in different circumstances. The motor homunculus is some form of representation of areas from which involuntary movements can be produced by artificial and passive means; is this the same homunculus that would be derived following a different form of cortical excitation such as magnetic stimulation, or when voluntary movements are generated? This contrasts with the sensory homunculus, which in Penfield's observations denotes areas from which subjectively reported sensations in the periphery are produced by antidromic rather than orthodromic stimulation. The sensations reported were of various types, and included tinglings, numbness, sensations of movement, and rarely pain, feelings of thickness, and other sensations.

This is of considerable importance, since the homunculus as represented by Penfield gives the impression of single motor and sensory maps. From experiments using electrical stimulation techniques in monkeys, however, it seems probable that the concept of simple somatotopic organisation is inadequate ${ }^{8}$ For the sensory homunculus, Merzenich et al provide evidence for multiple maps representing different functions, and there is evidence in non-human primates that there are as many as seven or more somatosensory areas, "most of which appear to constitute complete, orderly representations of the body surface or deep body tissues". ${ }^{10}$ Apart from differences that might result from different stimulation techniques, it may also be maps of cortical potentials evoked peripherally are not identical to maps of perceived sensations induced by direct cortical stimulation. Thus Penfield's homunculus is not only ambiguous in what exactly it does represent, but also gives an unjustified impression of cortical representation which is both single and established.

Moreover, not only were Penfield's data obtained using comparatively large-diameter stimulating electrodes in patients with brain disease, but different individuals, both normal and those with diseases of the nervous system, have very different cortical areas from which sensory and motor phenomena can be elicited. Penfield was aware not only of this individual variation but also that the effects of cortical stimulation could change after surgery. ${ }^{1}$ Whilst the immense plasticity of cortical maps in animals is established (for review, see Wall ${ }^{11}$ ), there are also several examples of plasticity in humans. Confirming observations in animals, patients with damage affecting somatosensory systems show evidence of marked somatotopic reorganisation at thalamic level. ${ }^{12}$ At cortical level, plasticity of maps of outputs of the human motor system has been demonstrated after amputation, paraplegia and hemispherectomy, and changes in sensorimotor representation have been demonstrated in blind braille readers (for review, see Pascual-Leone et $a l^{13}$ ). What of the homunculus in these patients?

Results of cortical stimulation in animals had of course been illustrated before, for instance by superimposing numbers and then words for the relevant part on drawings of the brain. ${ }^{14}$ Nevertheless, the first figurine was human, with Penfield's homunculus the precedent for the profusion of illustrations of experimental work on animals. Fundamental studies on cortical localisation using stimulation and evoked potential techniques, particularly by Bard, Woolsey et al, were illustrated in analogous fashion; the simiusculus, the cortical representation of the monkey, soon appeared, as did cortical representation of the rabbit, rat, cat and doubtless other animals (see Woolsey ${ }^{15}$ ). The pig and its large snout representation was another example illustrated in a different form by Adrian. ${ }^{16}$ Interesting, since it showed another form of 
physiological process is the figurine placed in the cerebellum by Fulton, ${ }^{17}$ and which he called a "primunculus" rather than homunculus. It illustrated the approximate distribution of excitatory and inhibitory foci in the anterior cerebellum. All these animal representations are more figurative than accurate, contrasting with the extremely accurate mapping of localised evoked potentials illustrated diagrammatically rather than by means of figurines. ${ }^{15}$

Even as a form of straightforward representation, the homunculus is curious. Just as the head has been the site of numerous fantastic yet symbolic compositions since Arcimboldo in the sixteenth century, ${ }^{18}$ so with the twentieth century homunculus there is artistic licence and embellishment. The border between fact and imagination has been blurred: the homunculus has been imbued with character, an attribute which many a viewer will experience, and which is perhaps unique in terms of scientific illustration. Thus the first homunculus drawn in 1937 is described by Penfield and Boldrey as "grotesque". . The later ones are "brought forth", and the drawings clearly have expressions and a personality. In 1937, the face is smiling broadly and is missing half its teeth; the 1950 versions show a wide-open mouth on the motor half of the homunculus, and a closed mouth and rather sad expression on the sensory half. ${ }^{2}$

It is tempting to dismiss this anthropomorphic development as an artistic whim or amusement. Whilst later relegating homunculi to the status of cartoons and aidesmemoires for students, ${ }^{23}$ at least initially the drawings were a serious endeavour, confirmed by comments such as that "certain inaccuracies in this figurine are corrected in the text"'-comments which preclude a frivolous intent. Not only was at least the first homunculus therefore a carefully thought out device, but as a consequence it could provoke strong reactions. Thus the concept of the homunculus was roundly castigated by Walshe: "even today cortical cartography is eagerly pursued as new modes of electrical stimulation uncover fragments of electrical excitability in new cortical territories. Nor are the moderns content with maps, for homunculi and simiusculi have now made their horrid appearances, lineal descendants of Lewis Carroll's Jabberwock, purporting to depict the fair face of nature, but in fact achieving something quite unnatural". ${ }^{19}$ It is ironic that Walshe, too, imbues the homunculi he so dislikes with personality, in this instance, horrid.

Noteworthy is Walshe's reference to cartography, since this alludes to one of the functions of such drawings: to represent the link between points on the surface of the body with areas within. The essence of all such drawings is that they are forms of maps, which represent how one collection of points is linked to another. ${ }^{20}$ Maps, however, are designed to be accurate as well as informative, and apart from the paucity of information available particularly in humans, there are formidable cartographic difficulties in using a device such as an homunculus as a type of map. These difficulties include two-dimensional representation of diverse, three-dimensional information both in the periphery and in the brain, the representation of multiple and sometimes overlapping images, and simultaneous representation of bilateral and unilateral data. The particular problems and potential misuse of mapping the electrical activity of the brain have been admirably reviewed by Binnie and MacGillivray. ${ }^{21}$

It is clear that both scientifically and graphically there is considerable ambiguity in what Penfield was intending to illustrate. This produces unease-even the preceding example of the map fails. Perhaps this is because the homunculus is a form of representation, and any representation of cerebral function is also ambiguous. The definition and nature of representation in science have never been satisfactorily defined. As Gooddy points out, "representation is at the bottom of so many schemes of neurological thinking, whether they be clinical or experimental". ${ }^{22}$ This is of considerable importance, not least since inappropriate representation could actually impede scientific advance. It might even be the homunculus is an instance of this. Gooddy's conclusion that "A function does not originate in a specific region of brain tissue, where its 'representation' has been traditionally located" is still valid today, but it points to the problem of how brain function can ever be illustrated. Amongst other aspects discussed above, it is this unresolvable difficulty which makes the homunculus an unsatisfactory and perhaps even misleading image.

The periphery of the homunculus is an additional aspect that requires comment. Penfield's homunculus is shown with an outline which encompasses the area of cortical representation. What exactly does this outline represent, for we do not feel the outline or periphery of our bodies? As Schilder comments, “...the outline of the skin is not felt as a smooth and straight surface. This outline is blurred. There are no sharp borderlines between the outside world and the body". ${ }^{23}$ The homunculus, however, is outlined by an imaginary 'envelope' which does not exist.

Another phenomenon where the border is blurred between fact (pathophysiology) and imagination (subjectivity) concerns phantom sensations. Some patients report that the phantom that follows amputation often fades, but it does so in a non-sequential manner. For a phantom limb, it has been stated that: "The sequence of disappearing parts follows, with the possible exception of the joints, the Penfield-Boldrey homunculus. Those parts which have large areas of representation on the homunculus...are the very same parts which have the longest phantom life. By contrast, those which have minimal representation on the homunculus are relatively short-lived as phantoms". ${ }^{24}$ Whilst this concept has been rejected for a number of reasons, ${ }^{25}$ it has to be accepted that newer theories are as tenuous as those that invoke the homunculus. It requires considerable ingenuity to attempt to describe a phantom phenomenon in terms of phantom representation.

It can scarcely have escaped Penfield's notice that both the term and the concept of the "homunculus" are old, dating at least since medieval times; they are also strange. The term merely means "little man" or "manikin", and again it is curious that Penfield and his collaborators should have used this term. A special use for the term seems implied, since, for example, a man portrayed on a street sign would not be designated a homunculus. Derived from the medieval idea of creating gold from base metals, the concept of the homunculus in the past embodied the idea of being able to create a diminutive human, a concept familiar to Paracelsus and later alchemists. Modern psychology has also embraced the homunculus-the concept of a little person within oneself, having a personal, internal role, and perhaps the inner person with whom one converses in internal speech: the function of this inner person is to provide an explanation or interpretation of the outside world-for example, an inner person that is responsive to one's pain, or to visual images, or other experiences. The inner person, however, explains nothing, and the usefulness of this concept is dubious. ${ }^{26}$

Even beyond the human and animal brain, the homunculus has acquired status, for it has actually been endowed with teleological attributes. Thus "The gross distortion of the imaginative 'little man' shows the high 


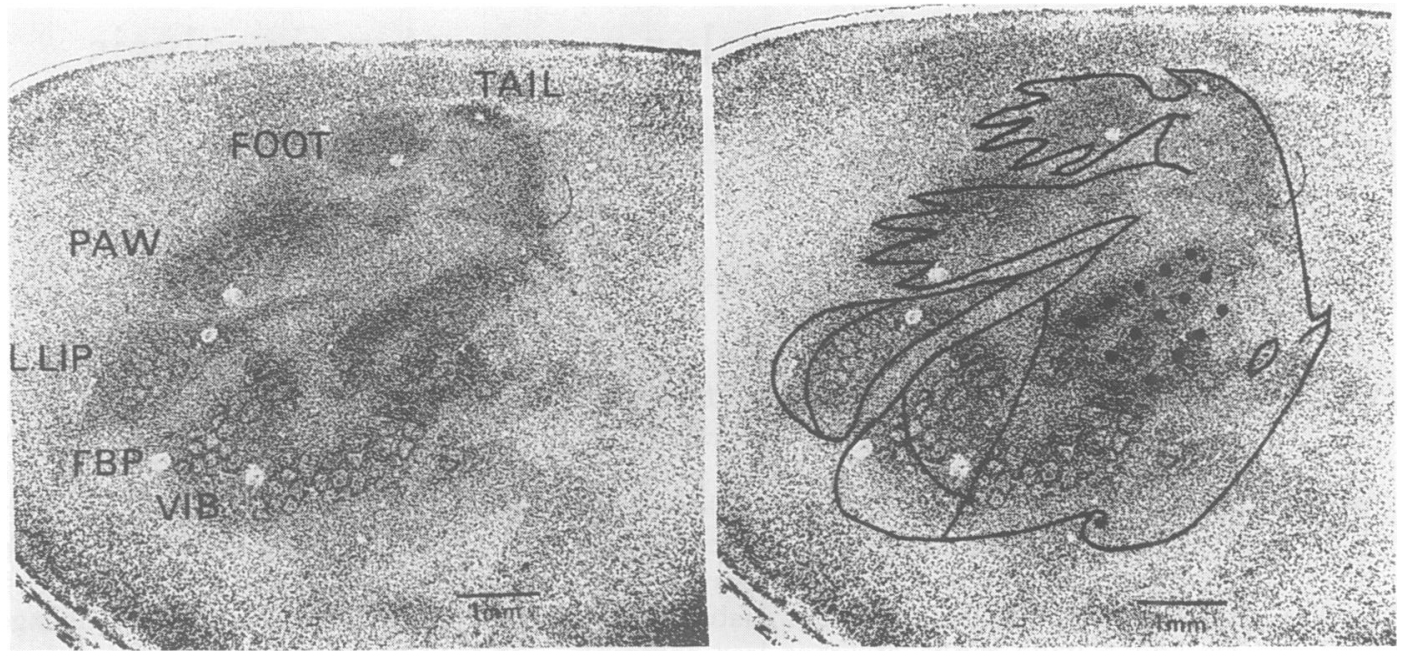

Figure 5 Photomicrograph showing the relationship between somatotopic subareas and architectural organisation of the rat sensory cortex, with parts of the body drawn around the cortical areas to which they project. Welker, 1976. Reprinted from fournal of Comparative Neurology 1976;166:173-190, with permission of The Wistar Institute Press.

priority that nature has placed on dexterity in the fingers and in the control of speech muscles. These two functional areas account for almost half the total area of the motor strip! A similar analysis in other mammals shows quite a different allocation of these areas, reflecting a different set of behavioural priorities". ${ }^{27}$ For the homunculus, science has now yielded to philosophy.

Penfield's homunculus was a deceptively simple and yet naïve concept. This type of illustration, a form of map, was a highly original attempt to portray graphically the observations of brilliant and painstaking research and one which has had a lasting influence as a mode of representation. It is memorable and useful. It has, however, been of limited and even doubtful scientific value, since fact and fancy have been confused. Illustration of brain function by projected drawings may best be reserved for those rare instances where true images can be derived and recorded. An example is the different cytoarchitectural subareas that can be discerned on photomicrographs of the rat sensory cortex. These disjunctive somatotopic areas, correlating with the projected peripheral body part, allow the different parts to be identified and drawn, and a sensory "rattunculus" can be derived (fig 5). ${ }^{28}$ Representation of everything else may best be served by an unambiguous diagram or words.

I am indebted to Dr P W Nathan and Dr D Fish for their most helpful comments, and Dr E Whitcombe for drawing $\mathrm{my}$ attention to the paper by Dykes and Ruest

The National Hospital for Neurology and Neurosurgery.

G D SCHOTT Que'en Square, London WC1N $3 B G, U K$

1 Penfield W, Boldrey E. Somatic motor and sensory representation in the cerebral cortex of man as studied by electrical stimulation. Brain 1937;60:389-443.

2 Penfield $\mathbf{W}$, Rasmussen T. The cerebral cortex of man. New York: Macmillan, 1950:44, 56, 214-15.

3 Penfield W, Jasper H. Epilepsy and the functional anatomy of the human brain. London: J and A Churchill, 1954:105-106; 159

4 Bates JAV. Stimulation of the medial surface of the human cerebral hemisphere after hemispherectomy. Brain 1953;76:405-47.
5 Hassler R, Mundinger F, Riechert T. Stereotaxis in Parkinson syndrome. Berlin: Springer Verlag, 1979: (front cover).

6 Creutzfeldt OD. Cortex cerebri. Berlin: Springer Verlag, 1983: 216.

7 Cull P. Medical Illustration. In: Jennings S, ed. The new guide to professional illustration and design. London: Headline, 1987: 40-43.

8 Gould HJ, Cusick CG, Pons TP, Kaas JH. The relationship of corpus callosum connections to electrical stimulation maps of motor, supplementary motor, and frontal eye fields in owl monkeys. $\mathcal{f}$ Comparative Neurology 1986;247:297-325.

9 Merzenich MM, Kaas JH, Sur M, Lin C-S. Double representation of the body surface within cytoarchitectonic areas $3 \mathrm{~b}$ and 1 in "Sl" in the ow monkey (Aotus trivirgatus). $\mathcal{f}$ Comparative Neurology 1978;181:41-74.

10 Merzenich MM, Kaas JH. Principles of organization of sensory-perceptual systems in mammals. Progr Psychobiol Physiol Psychol 1980;9:1-42.

11 Wall JT. Variable organization in cortical maps of the skin as an indication of the lifelong adaptive capacities of circuits in the mammalian brain. of the lifelong adaptive capacities

12 Lenz FA. The thalamus and central pain syndromes: human and animal studies. In: Casey KL, ed. Pain and central nervous system disease: the central pain syndromes. New York: Raven Press, 1991:171-182.

13 Pascual-Leone A, Cohen LG, Hallett M. Cortical map plasticity in humans. Trends Neurosci 1992;15:13-14.

14 Leyton ASF, Sherrington CS. Observations on the excitable cortex of the chimpanzee, orang-utan and gorilla. Q f Exper Physiol 1917;11:135-222.

15 Woolsey CN. Organization of somatic sensory and motor areas of the cerebral cortex. In: Harlow HF, Woolsey CN, eds. Biological and biochemical bases of behavior. Madison: The University of $\mathrm{W}$ isconsin Press, 1958:63-81.

16 Adrian ED. Motor and sensory areas of the brain. The physical background of perception. Oxford: Oxford University Press, 1946:31-47.

17 Fulton JF. Functional localization in the frontal lobes and cerebellum. London: Oxford University Press, 1949:129-130.

18 Kaufmann TD. The allegories and their meaning. In: Hulten P, ed. The Arcimboldo effect. Milan: Bompiani, 1987:89-109.

19 Walshe FMR. Some reflections upon the opening phase of the physiology of the cerebral cortex, 1850-1900. In: Poynter FNL, ed. The history and philosophy of knowledge of the brain and its functions. Amsterdam: B. M. Israel, 1973:223-4.

20 Dykes RW, Ruest A. What makes a map in somatosensory cortex? In: Jones, EG, Peters A, eds. Cerebral cortex, Vol 5, Sensory-motor areas and aspects of cortical connectivity. New York: Plenum, 1986:1-29.

21 Binnie CD, MacGillivray BB. Brain mapping - a useful tool or dangerous toy? I Neurol Neurosurg Psychiatry 1992;55:527-9.

22 Gooddy W. Cerebral representation. Brain 1956;79:167-87. Paul, Trench, Trubner, 1935:85.

24 Simmel ML. On phantom limbs. Arch Neurol Psychiatry, (Chicago) $1956 ; 75,637-47$.

25 Melzack R. Phantom limbs, the self and the brain. Canadian Psychology 1989;30,1-16.

26 Dennett DC. Brainstorms. Philosophical essay's on mind and psychology Hassocks, Sussex: Harvester Press 1979:57-59.

27 Pugh GE. The biological origin of human values. London: Routledge and Kegan Paul, 1978:140.

28 Welker C. Receptive fields of barrels in the somatosensory neocortex of the rat. $f$ Comparative Neurol 1976;166:173-190. 